\title{
A NEW HYPERBOLIC TANGENT MODELLING APPROACH FOR THE CREEP BEHAVIOUR OF THE SINGLE CRYSTAL NICKEL-BASED SUPERALLOY CMSX4
}

\author{
H. Basoalto ${ }^{1}$, B. Vermeulen ${ }^{1}$, J.W. Brooks ${ }^{1}$, G. Coventry ${ }^{2}$, S. Williams ${ }^{2}$, J. Mason-Flucke ${ }^{3}$ and S. Bagnall ${ }^{3}$ \\ ${ }^{1}$ QinetiQ Ltd, Farnborough, GU14 0LX, UK \\ ${ }^{2}$ Rolls-Royce plc, Derby DE24 8BJ, UK \\ ${ }^{3}$ Rolls-Royce plc, Bristol BS34 7QE, UK
}

Keywords: Single crystals, CMSX4, Creep, microstructure-explicit modelling, CT07

\begin{abstract}
Rolls-Royce has developed a hyperbolic tangent representation of creep, referred to as CT07 [1], which explicitly relates the accumulated creep strain to the current time, stress and temperature. Although, such an approach has been successfully applied to a number of nickel-based superalloys, it will be shown that it does not adequately capture the shapes of the creep curves and rupture times of the single crystal CMSX4 at temperatures below $800^{\circ} \mathrm{C}$ and above $1000^{\circ} \mathrm{C}$. This result arises from the implicit assumption made in CT07 that the generic shape of the creep curve is tertiary dominated, which is not observed in CMSX4. This paper presents a new hyperbolic tangent formulation that accounts for sigmoidal creep, the large primary creep strains observed at low temperatures, the tertiary dominated creep behaviour at high temperatures $\left(850-1000^{\circ} \mathrm{C}\right)$ and the secondary creep dominated response above $1000^{\circ} \mathrm{C}$. A microstructure-explicit creep model has been used to derive parameters, such as the incubation time at low temperatures, which feed into the new hyperbolic tangent formulation. It will be shown that the new hyperbolic tangent model predictions of the CMSX4 creep response are in good agreement with the experimental data over a wide range of stresses and temperatures.
\end{abstract}

\section{Introduction}

The anisotropic high temperature creep behaviour of single crystal superalloys is a complex function of the underlying crystallography and microstructure, as well as, the applied stress and temperature. The single crystal alloy CMSX4 is a second generation nickel-based superalloy with up to $70 \%$ volume fraction of the intermetallic $\gamma^{\prime}$ strengthening phase, which is currently being used by Rolls-Royce in the production of high pressure turbine blades. The ability to model and predict accurately the behaviour of such an alloy in service is an important step in improving current design and lifing procedures of gas turbine blade components.

The aim of the paper is to present a uniaxial $<001>$ creep model for the single crystal superalloy CMSX4. The starting point for the model development is the Rolls-Royce hyperbolic tangent representation of creep strain, otherwise known as CT07 [1]. In this representation of creep the starting point is the relationship between the applied stress and the accumulated strain at a given time slice and temperature. This relationship between the applied stress, $\sigma$, and the accumulated strain, $\varepsilon$, is assumed to have the form,

$$
\sigma=\sigma_{i}\left[1+\tanh \left(k \ln \left(\varepsilon / \varepsilon_{i}\right)\right)\right]
$$

where $\sigma$ and $\varepsilon_{i}$ are functions of temperature and time. The $\sigma_{i}$ parameter is equal to half the ultimate tensile strength (UTS), $\varepsilon_{i}$ is equal to the strain at the point of minimum strain rate when the applied stress equals $\sigma_{i}$, and $k$ a free fitting parameter, discussed in detail later in the paper. The parameters are determined by numerical fit to the creep database, which are then used to calculate a creep curve by rearranging Equation (1),

$$
\varepsilon=\varepsilon_{i} \exp \left(\frac{1}{2 k} \log _{10}\left[\sigma /\left(2 \sigma_{i}-\sigma\right)\right]\right)
$$

In the last expression the creep strain becomes singular as the applied stress approaches the UTS of the material, i.e., when $\sigma / \sigma_{i} \rightarrow 2$. This is not mirrored in real materials, as a microstructural instability (for example, the formation of micro-cavities) will result in a finite rupture strain. However, the fitting procedure for the material parameters ensures that the singularity occurs outside the usable range of the model [2].

At present, an important advantage of using a model that assumes an explicit relationship between the accumulated creep strain and time is that implementation of such a model in a finite element code is computationally less costly compared to more sophisticated models where the deformation behaviour is tracked via a number of state variables that need to be updated at the end of the time increment. The strain-time trajectories given by Equation (2) have a 'generic shape' that is independent of temperature and stress. In the following section, it will be shown that this inherent property of the current hyperbolic tangent representation, although shown to be successful in a number of superalloys [1], puts serious limitations on modelling creep behaviour of the single crystal CMSX4. Even so, Basoalto [3] has shown that the CT07 creep model is able to capture the creep response of this alloy at intermediate temperatures ranging from $850-1000^{\circ} \mathrm{C}$. For temperatures outside this range the creep curves of CMSX4 do not have the classical shape assumed in CT07. This provided the main driving force for the work presented in this paper, i.e., to extend the current Rolls-Royce creep model to single crystal superalloys. An overview of the uniaxial $<001>$ creep behaviour will be given in Section 2.1, which will serve to identify the necessary requirements that any new model (based on a hyperbolic tangent representation) must satisfy in order to accurately describe the high temperature behaviour of the single crystal CMSX4.

\section{Material Behaviour and Model Requirements}

This section focuses on the main micromechanism believed to effect or drive dislocation creep in CMSX4. It is not intended to serve as a complete treaty on the subject, but instead to provide justification for a new hyperbolic tangent based modelling approach to single crystal superalloys.

\section{Creep Curves}

Over the last decade or so a number of micromechanisms have been reported in the scientific literature that directly 
affects the creep behaviour of the single crystal nickel-based superalloy CMSX4 [4-9]. These mechanisms include microstructural changes resulting from thermodynamic instabilities, as well as, athermal effects such as dislocation cutting of the $\gamma^{\prime}$ particles. In general, these processes result in an increased creep rate and therefore shorter lifetimes. Ashby and Dyson [10] have referred to such processes as forms of damage, in order to bring microstructural re-arrangements into a continuum damage mechanics framework. The operation of such micromechanisms is a function of stress and temperature, resulting in the strain-time trajectories having very different shapes between 650 and $1200^{\circ} \mathrm{C}$.

For temperatures $\leq 800^{\circ} \mathrm{C}$, relatively high stresses are required to produce significant creep rates. The strain-time trajectories are characterised by a period of near zero creep activity, referred to as the 'incubation period'. This can be tens to hundreds of hours depending on the stress and temperature. Pollock and Argon [8] proposed that this low creep activity is a reflection of the low initial dislocation densities that exist in these materials resulting in a 'waiting' transient as the dislocation density builds up to produce measurable strain rate. Primary creep then follows with creep strains ranging from $20-30 \%$ at $650^{\circ} \mathrm{C}$ for stresses $>800 \mathrm{MPa}$ and $4-8 \%$ at $750^{\circ} \mathrm{C}$ for stresses $>650 \mathrm{MPa}$, before the creep rate reaches a minimum. Shearing of the $\gamma^{\prime}$ particles by dislocations is believed to play an important role at low temperatures and high stresses [6, 9].

At intermediate temperatures, defined here as those $>800^{\circ} \mathrm{C}$ and $\leq 950^{\circ} \mathrm{C}$, dislocation activity is mainly confined to the $\gamma$ channels for the stress levels of interest, giving rise to deformation curves that are tertiary creep dominated with small primary creep transients (Figure 1).

For temperatures above $950^{\circ} \mathrm{C}$, the deformation curves are observed to go from tertiary creep dominated shapes to a creep response that is clearly 'secondary creep controlled', see Figure 2. In this temperature range the $\gamma^{\prime}$ particles undergo a morphological change from initially cuboidal particles to a plate-like or rafted structure. Since CMSX4 is a negative misfit alloy, the $\gamma^{\prime}$ rafts form perpendicular to the loading axis. This transformation severely restricts dislocation movement resulting in a reduction in the measured creep strain rates. For lack of a better term, the phrase 'secondary controlled' transient will be used to describe this situation, and is not to be confused with the classical use of the phrase to describe a steady state dislocation hardening-recovery process. The subsequent dislocation density build-up gives rise to pile-up stresses against $\gamma^{\prime}$ rafts, which eventually cut through the rafted structure resulting in a sharp tertiary transient.

\section{Model Requirements}

From observations of the creep response between $650-1200^{\circ} \mathrm{C}$ and over a stress range between $25-900 \mathrm{MPa}$, the following requirements need to be satisfied by any model trying to predict the creep behaviour of single crystal nickel-based superalloy CMSX4 <001>:

1. Sigmoidal creep. The model must be able to capture the low strain activity observed at low temperatures $\left(\leq 800^{\circ} \mathrm{C}\right)$ resulting in an incubation period;

2. The model must be able to capture the large creep strains accumulated at the end of the primary creep transient;
3. For temperatures in excess of $1000^{\circ} \mathrm{C}$, the model must be able to account for the steady creep strain rate behaviour that dominates most of the life.

The following section outlines the general mathematical modifications of the CT07 creep model, in order to satisfy the above modelling requirements.

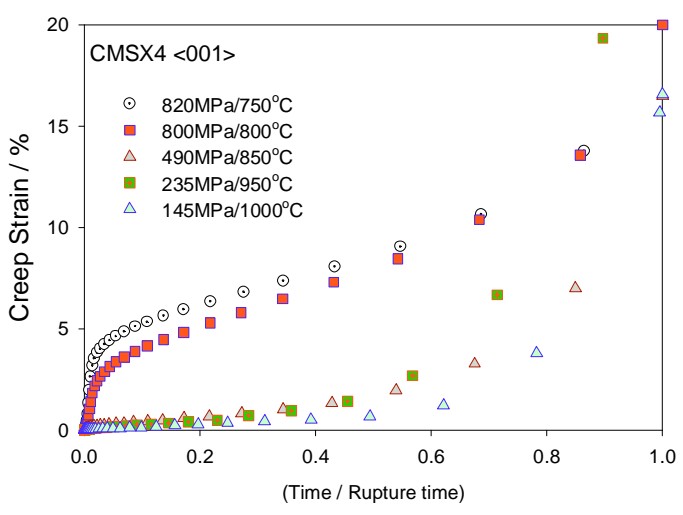

Figure 1 Normalised CMSX4 <001> creep curves for a number of stress and temperature conditions.

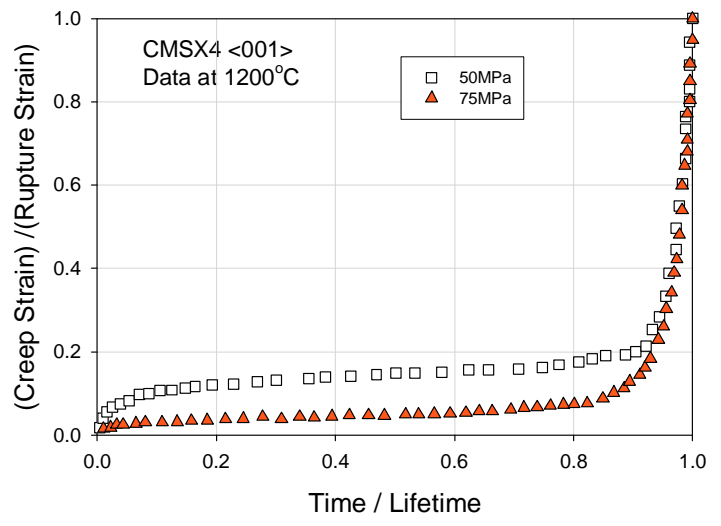

Figure 2 CMSX4 $<001>$ creep behaviour at $1200^{\circ} \mathrm{C}$.

\section{Single Crystal Hyperbolic Tangent Representation}

A new hyperbolic tangent representation is introduced [11]:

$$
\varepsilon=\lambda(t, \sigma, T) \varepsilon_{i}(t, \sigma, T) \exp \left(\frac{\log _{10}\left[\sigma /\left(2 \sigma_{i}-\sigma\right)\right]}{2 k(T)}\right) .
$$

In this form, the original hyperbolic tangent representation has been modified in three fundamental ways:

- A $\lambda$-multiplier is introduced. This multiplier is essential for modelling the sigmoidal creep behaviour observed at low temperatures. The $\lambda$-multiplier is defined in such a way that its value varies from 0 to 1 over an incubation period and has the following form,

$$
\lambda(t, \sigma, T)=\frac{1}{2}\left[1+\tanh \left(G(\sigma, T)\left(t-t_{0}(\sigma, T)\right)\right)\right],
$$

where $G(\sigma, T)$ and $t_{0}(\sigma, T)$ are functions of stress and temperature. A physical interpretation of the parameter $t_{0}(\sigma, T)$ is that it represents the incubation period; 
- A stress dependent $\varepsilon_{i}$ is introduced in Equation (3) to model the high primary creep strains in the low temperature (high stress) regime;

- A new temperature dependency is introduced through the $k$ parameter in Equation (3) to capture the creep curve shape above $1000^{\circ} \mathrm{C}$.

\section{Determining the Incubation Period, $t_{0}$}

As outlined in the previous section, sigmoidal behaviour can be introduced within a hyperbolic tangent representation of creep by defining a time-stress-temperature dependent multiplier, $\lambda$, that varies between zero and unity over a time length scale equal to the incubation period. The stresstemperature dependence of the multiplier is embedded within the incubation time parameter, $t_{0}$, and the gradient, $G$, of the multiplier evaluated at $t=t_{0}$, see Equation (4). A dislocation density evolution modelling approach proposed by Dyson and co-workers [12-15] has been used to capture the stress and temperature dependencies of the sigmoidal parameters.

Let the mobile dislocation density be denoted by $\rho_{m}$. It is assumed that the mobile dislocation network is made up of a gliding population, $\rho_{g}$, which gives rise to the macro-strain rate, and a trapped dislocation network, $\rho_{c}$. Assuming that the net dislocation content in a unit volume of material can be modelled by a dislocation generation-trapping model, then the basic governing equation for the net flux of dislocation segments can be expressed as [12],

$$
\frac{\partial \rho_{g}}{\partial t}=\dot{\rho}_{g}^{(+)}\left(\rho_{m}, \sigma, T\right)-\rho_{m} \frac{\bar{v}_{g}}{L_{p}},
$$

where $\dot{\rho}_{m}^{(+)}\left(\rho_{m}, \sigma, T\right)$ is the rate of dislocation generation, whose details depend on the nature of the dislocation-obstacle interaction. The second quantity on the right hand side of Equation (5) is a trapping term, which describes the rate at which gliding dislocation segments are trapped by obstacles after travelling a mean distance, $L_{p}$, taken to be the interparticle spacing, and an average glide velocity $\bar{v}_{g}$. It can be shown that the basic solution to Equation (5) has the following form,

$$
\begin{gathered}
\rho_{m} \bar{v}_{g}=\dot{\rho}_{m}^{(+)}\left(\rho_{m}, \sigma, T\right) L_{p} j(t) \\
j(t)=\left(1-\exp \left(-\frac{\bar{v}_{g}}{d_{m f p}}\right) t\right)
\end{gathered}
$$

Combining the last result with the Orowan-Bailey relation, the creep strain rate, $\dot{\varepsilon}$, can be expressed as [12-16],

$$
\dot{\varepsilon}=b \rho_{m} \bar{v}_{g}=\frac{b \dot{\rho}_{m}^{(+)}\left(\rho_{m}, \sigma, T\right) L_{p}}{M} j(t),
$$

where $b$ is the Burger's vector and $M$ is the Taylor factor. Figure 3 shows a schematic diagram of the creep strain evolution predicted by Equation (8). For most cases the exponential term in $j(t)$ (Equation (7)) decays very quickly and is usually ignored, so that the steady state creep rate is,

$$
\dot{\varepsilon}_{s s}=\frac{b \dot{\rho}_{m}^{(+)}\left(\rho_{m}, \sigma, T\right) L_{p}}{M} .
$$

For engineering precipitate strengthened materials an internal back stress develops from stress partitioning between a creeping matrix and elastically deforming particles. This is the origin of primary creep in these materials, which is not based on the dislocation-dislocation hardening-recovery equilibrium configuration that holds true for pure metals or simple alloys.
Let us assume that for high volume fraction nickel based superalloys the exponential term appearing in Equation (7) does not decay quickly. This term will vary between zero and unity. Re-arranging for time, $t$, and equating this time to the incubation parameter $t_{0}$, yields,

$$
t_{0}=\frac{L_{p}}{\bar{v}_{g}} \ln \left(\frac{1}{1-j^{*}}\right)=k \frac{L_{p}}{\bar{v}_{g}} .
$$

Basoalto and Dyson [16] have calculated an expression for the generation rate, $\dot{\rho}_{m}^{(+)}\left(\rho_{m}, \sigma, T\right)$, for the case of dislocations moving through a field of precipitates. They show that for a unimodal particle dispersion the macro-strain rate has the form,

$$
\begin{aligned}
& \dot{\varepsilon}=\dot{\varepsilon}_{0} \exp \left(-\frac{Q_{d / j}}{R T}\right) \sinh \left(\frac{\sigma}{\sigma_{0}}\right) \\
& \dot{\varepsilon}_{0}=\frac{2}{M} \rho_{m} \phi_{p}\left(1-\phi_{p}\right) \frac{L_{p}}{r_{p}} c_{j, 0} D_{v, 0} \\
& \sigma_{0}=\frac{M k T}{b^{2} L_{p}} K\left(\phi_{p}\right)
\end{aligned}
$$

where $\phi_{p}$ and $r_{p}$ are the volume fraction and radius of the particulate dispersion, $D_{v, 0}$ is the self-diffusion coefficient, $c_{j, 0}$ the jog density coefficient, $Q_{d, j}$ the activation energy, and $R$ the gas constant. The strength stress parameter, $\sigma_{0}$, is a function of the inter-particle spacing, which in turn makes it a function of the volume fraction and particle radius of the second phase.

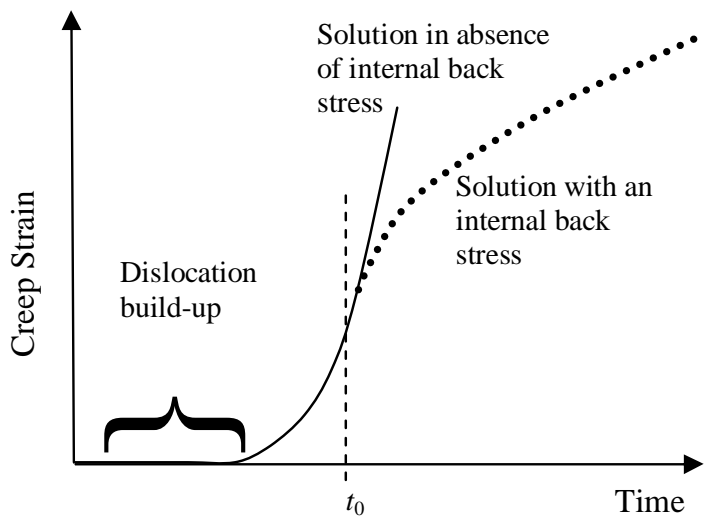

Figure 3 Dislocation build-up model and sigmoidal creep.

It follows from Equation (10) that in order to calculate the incubation time, $t_{0}$, the average dislocation glide velocity, $\bar{v}_{g}$, needs be determined. By combining Equations (11) with the Orowan-Bailey relation the dislocation glide velocity can be calculated,

$$
\begin{aligned}
& b \beta \rho_{m} \bar{v}_{g}=\rho_{m} c_{j, 0} D_{v, 0} \phi_{p}\left(1-\phi_{p}\right) \frac{L_{p}}{r_{p}} \exp \left(-\frac{Q_{d, j}}{R T}\right) \sinh \left(\frac{\sigma}{\sigma_{0}}\right), \\
\Rightarrow & \bar{v}_{g}=\frac{c_{j, 0} D_{v, 0} \phi_{p}\left(1-\phi_{p}\right)}{b \beta} \frac{L_{p}}{r_{p}} \exp \left(-\frac{Q_{d, j}}{R T}\right) \sinh \left(\frac{\sigma}{\sigma_{0}}\right),
\end{aligned}
$$

where $\beta$ is a numerical factor relating the gliding dislocation density to the total mobile density, i.e., $\rho_{g}=\beta \rho_{m}$. Putting this expression for the glide velocity into (10), gives the following form for the incubation period,

$$
t_{0}=\frac{b \beta k}{c_{j, 0} D_{v, 0} \phi_{p}\left(1-\phi_{p}\right) \exp \left(-\frac{Q_{d, j}}{R T}\right) \sinh \left(\frac{\sigma}{\sigma_{0}}\right)} .
$$


Thereby, yielding the following theoretical relationship for the incubation period as a function of stress and temperature,

$$
t_{0} \exp \left(-\frac{Q_{d / j}}{R T}\right)=C^{*}\left(\phi_{p}\right) / \sinh \left(\sigma / \sigma_{0}\right)
$$

where $C^{*}\left(\phi_{p}\right)$ is a function of volume fraction of the dispersed phase, which is discussed later in detail. Note that Equation (16) predicts all measured values of $t_{0}$, and can be made to collapse onto a single master curve.

As already stated, 'measured' values for $t_{0}$ were obtained by fitting Equations (2) and (3) to creep curves possessing sigmoidal creep behaviour. Figure 4 shows the stress dependence of the 'measured' left hand side of Equation (16), with all the data collapsing onto a single curve, as suggested by the theory. Equation (16) was fitted to the data with an activation energy of $320 \mathrm{kJmol}^{-1}$.

Assuming a stable microstructure the microstructure-explicit model gives a $\sigma_{0}$ at $750^{\circ} \mathrm{C}$ that is too large and does not capture the incubation period in the low temperature and high stress regime (dashed line in Figure 4). However, in reality such a simplifying assumption can not satisfied since a superalloys microstructure will not remain static during high temperature deformation. According to the microstructure explicit creep model of Basoalto et al. [14], $\sigma_{0}$ is a function of the volume fraction of the $\gamma^{\prime}$ particles, which decreases with increasing temperature and vanishes at the $\gamma^{\prime}$ solvus. In addition, as the stress increases more dislocations can overcome the $\gamma^{\prime}$ particles by cutting or Orowan by-passing, which results in a decrease of the $\sigma_{0}$ parameter at high stresses. Hence, $\sigma_{0}$ will vary in general from an upper bound value when the particles are stable and able to effectively resist dislocation motion and a lower bound associated with a less effective particle dispersion in preventing dislocation motion. Since it is not the purpose of this work to develop a fully microstructure-explicit creep model for single crystal superalloys, this paper will not explore such explicit dependencies. However, it was found that the following expression captures these stress effects,

$$
\sigma_{0}[\sigma]=\sigma_{0, R}\left[1-\alpha \tanh \left(\frac{\sigma-s_{1}}{s_{2}}\right)\right]
$$

where $\sigma_{0, R}, s_{1}, s_{2}$ are fitting parameters. Equations (16) and (17) were fitted to the data and are presented in Figure 4 (solid line).

Figure 5 shows the actual fits of the incubation times to the measured values for a number of temperatures. The proposed stress-temperature representation captures the data well at the low temperature and high temperature regime. However, at intermediate temperatures, Equations (16) and (17) predict $t_{0}$ values of several hours, which is not observed in the data since no sigmoidal creep is present. This shortfall is attributed to the fact that within the current model development the temperature effects on the strength parameter, $\sigma_{0}$, have not been accounted for so far. Note that such a dependency can be calculated from theory, however, in order to be consistent with the approach of the work presented, a temperature correction is introduced such that for temperatures $>820^{\circ} \mathrm{C}$ no incubation is observed. This correction is proposed by modifying the $C^{*}$ parameter appearing in Equation (16) as

$$
C^{*}\left(\phi_{p}\right)=C^{*}=\frac{1}{2} C_{0}^{*}\left[1-\tanh \left(\frac{T-T^{*}}{T_{n}}\right)\right]
$$

where $C_{0}^{*}, T^{*}, T_{n}$ are fitting constants, which are reported in [11].

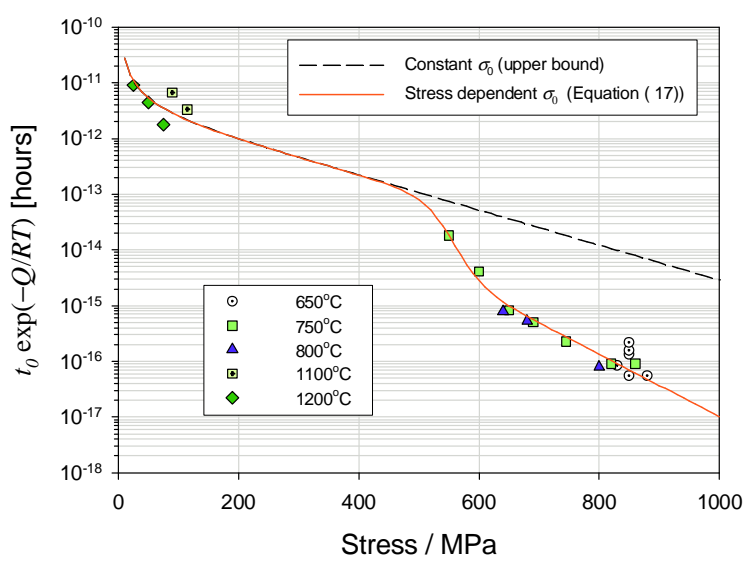

Figure 4 Fitted incubation parameter values versus stress. Also shown is the model fit given by Equation (16) together with lower and upper bound fits.

\section{Determination of $G(\sigma, T)$}

From Equation (4), the $G(\sigma, T)$ parameter is the gradient of the $\lambda$-multiplier at $t=t_{0}{ }^{1}$. Consequently, from the definition of the creep strain within the new CT07 framework (Equations (3) and (4)) it follows that $G(\sigma, T)$ is proportional to the creep strain rate, $\dot{\varepsilon}$, at $t=t_{0}$, i.e.,

$$
G(\sigma, T) \propto \dot{\varepsilon}
$$

From Equation (3) the strain rate, $\dot{\varepsilon}$, can be re-written as,

$$
\begin{aligned}
G(\sigma, T) & \propto \dot{\varepsilon}_{0} \exp \left(-\frac{Q}{R T}\right) \sinh \left(\frac{\sigma}{\sigma_{0}}\right), \\
\Rightarrow \quad G(\sigma, T) & =h^{*} \exp \left(-\frac{Q}{R T}\right) \sinh \left(\frac{\sigma}{\sigma_{0}}\right)
\end{aligned}
$$

where $h^{*}$ is a rate constant of proportionality. Combining Equations (18) and (22) results in the following expression for the sigmoidal $G(\sigma, T)$ parameter,

$$
G(\sigma, T)=\frac{h^{*} C^{*}}{t_{0}}
$$

where $C^{*}$ is given by Equation (18). Figure 7 shows the best fits obtained using Equation (21) with the incubation representation presented in the previous section and an $h^{*}=$ $7 \times 10^{12} \mathrm{~s}^{-1}$.

\section{High Temperature Behaviour $\left(>1000^{\circ} \mathrm{C}\right)$}

The $k$-parameter appearing in Equation (3) was found to have a strong effect on the length of the secondary transient. In the original CT07 creep model this parameter is a constant.

$$
k=C A L K\left(\left(T_{M}-T\right) / \sigma_{i}\right)^{\text {Pow }}
$$

where $P O W$ and $C A L K$ are constants that are obtained by fitting the CT07 creep model to the CMSX4 <001> creep database. The remaining parameter $T_{M}$ is melting the temperature. However, numerical fitting of the new hyperbolic tangent equations to the CMSX4 <001> creep database revealed that the $k$ dependency in temperature had to be modified from that given by Equation (22), by introducing a temperature dependence through the $C A L K$ parameter [6].

\footnotetext{
${ }^{1}$ Actually, according to Equation (4) $\dot{\lambda}=G / 2$.
} 
Figure 6 shows the average of the fitted $C A L K$ values from $650-1200^{\circ} \mathrm{C}$. It can be seen that as the temperature increases from $650^{\circ} \mathrm{C}$ that CALK drops to a minimum at about $950^{\circ} \mathrm{C}$ before increasing again.

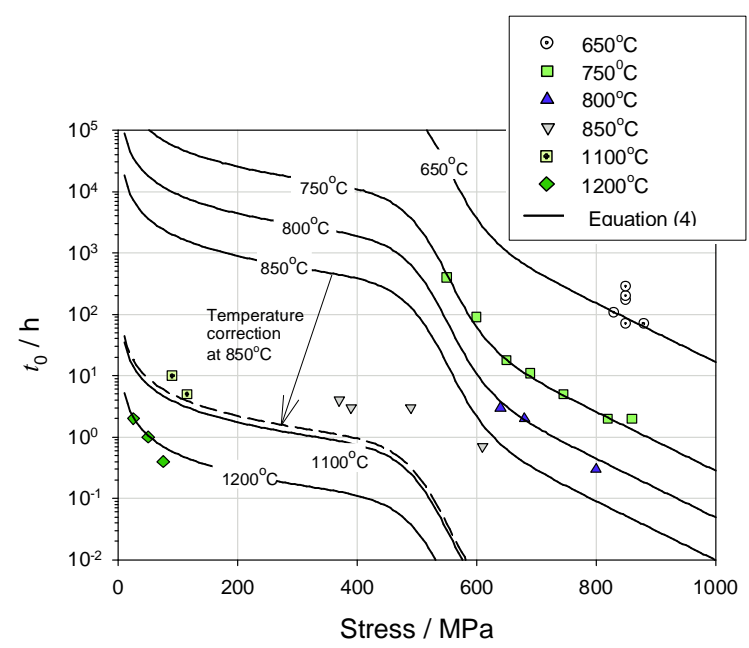

Figure 5 Measured incubation times and model fits. Note that there is insufficient data at $850^{\circ} \mathrm{C}$ to allow a proper fit of the incubation time parameter at this temperature.

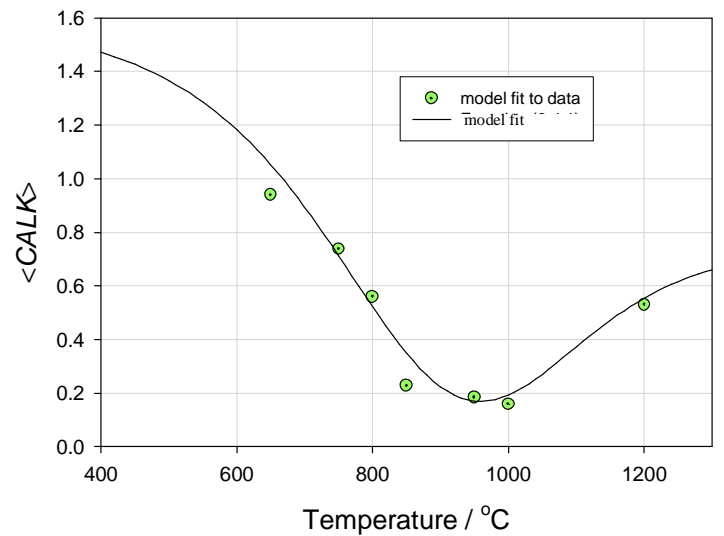

Figure 6 Temperature dependence of fitted $C A L K$ parameter.

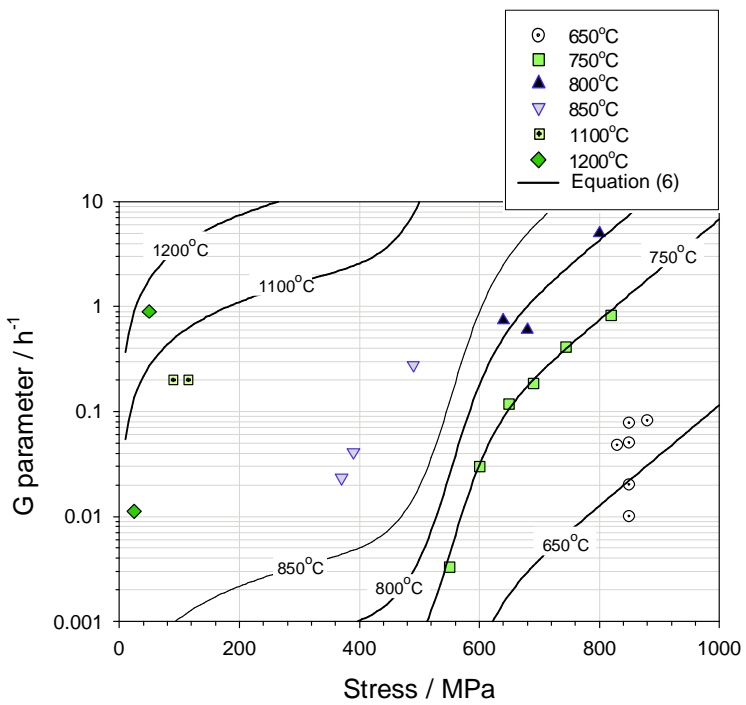

Figure 7 Stress-temperature dependence of the $G$ parameter.

\section{Model predictions}

Model predictions for the time to reach a number of strain levels $(0.1,0.2,5$ and $20 \%)$ as a function of stress at $750^{\circ} \mathrm{C}$ are presented in Figure 8 and are compared to experimental data. Also, shown are the stress-time contours generated by CT07 at 0.1 and $20 \%$ creep strain. It can be seen that the new hyperbolic tangent model captures the steep upturn in the curves at high stresses end associated with primary creep, which Equation (1) fails to capture. As the strain level increases, the stress-time contours shift to the right and change shape. Figure 9 shows measured strain-time trajectories for two stress levels at $750^{\circ} \mathrm{C}$ together with model predictions generated by CT07 and the new hyperbolic tangent representation. The proposed model framework predicts strain-time trajectories that are in good agreement with the experimental data, capturing the initial incubation transient, the extent of primary creep strain accumulation and the lifetimes.

Figure 10 shows data and model predictions for creep conditions of $115 \mathrm{MPa}$ at $1100^{\circ} \mathrm{C}$ and it can be seen that the new model predicts a creep response that is more consistent with the experimental data than that calculated using the original model formulation. Capturing the dominant secondary transient observed in CMSX4 in this temperature regime was the motivation for the modification to the stress function appearing in Equation (3), i.e., the modifications to the $k$-parameter described in the previous section.

\section{Conclusions}

- A new hyperbolic tangent representation of creep has been developed that accurately captures the high temperature <001> creep behaviour of the nickel-based single crystal superalloy CMSX4.

- Although, the current hyperbolic tangent representation used by Rolls-Royce has been successfully applied to a number of nickel-based superalloys, modifications are required to capture the shape of the $\langle 001\rangle$ uniaxial creep response of CMSX4 at temperatures below $800^{\circ} \mathrm{C}$ and above $1000^{\circ} \mathrm{C}$. This arises from the implicit assumption that creep behaviour can be represented by a conventional primary/secondary/tertiary creep curve shape with consistent trends across the required temperature range, which is not appropriate for CMSX-4. For this superalloy a number of micromechanisms (dislocation generation in the $\gamma$ channels, morphological changes and cutting of the $\gamma^{\prime}$ particles) are known to operate that influence its creep and plastic behaviour.

- Using current metallurgical understanding of the dominant micromechanisms operating in CMSX4 a number of model requirements have been identified to extend the hyperbolic tangent creep model to single crystals. The proposed mathematical representation is given by Equations (4) and (5), which account for the sigmoidal creep and high primary creep strains observed at low temperatures, as well as, handling the response at temperatures above $1000^{\circ} \mathrm{C}$.

- Using a microstructure-explicit modelling approach [1216], a functional form for the incubation time as a function of stress and temperature has been determined. The theoretical development is based on the assumption that the sigmoidal creep transient observed at low temperatures is due to a build-up of dislocation in the $\gamma$ channels [8]. 
- The new hyperbolic tangent representation for single crystal superalloys has been shown to capture the <001> creep behaviour of CMSX4 between $650-1200^{\circ} \mathrm{C}$ for a range of stresses of practical interest.

\section{References}

1. Williams, S.J., "An automatic techinique for the analysis of stress rupture data", Report MFR30017, Rolls-Royce plc, 1994.

2. Williams, S.J., "An automatic techinique for the analysis of creep strain data", Report MFR30018, Rolls-Royce plc, 1994.

3. Basoalto, H.C., "ATAP10: CMSX4 Isotropic Creep Model Programme Work Package 1", Report QINETIQ/FST/STT/CR052118, 2005.

4. Shollock, B.A., Ardakani, A., Ghosh, R.N., Basoalto, H.C. and McLean, M., "Deformation micromechanisms and anisotropic creep modelling of single crystal superalloys", in: Proceedings of Plasticity '99: Constitutive and Damage Modelling of Inelastic and Phase Transformation, Ed. Akhtar S. Khan, p. 193, 1999.

5. Nabarro, F.R.N., Cress, C.M. and Kotschy, P., "The thermodynamic driving force for rafting in superalloys", Acta Metal. Mater. 44, 3189, 1996.

6. Ardakani, M.G., Ghosh, R.N., Brien, V., Shollock, B.A. and McLean, M., "Implications of dislocation micromechanisms for changes in orienation and shape of single crystal superalloys", Scripta Mater., Vol. 39, Nos. 4/5, 465-472, 1998.

7. Ohashi, T., Hidaka, K. and Imano, S., "Quantitative study of the plastic slip deformation and formation of internal stresses in Ni-base superalloys", Mat. Sci. Eng., A238, 42-49, 1997.

8. Pollock,T.M. and Argon, A.S., "Creep resistence of CMSX3 nickel-based superalloy single crystal", Acta Metall. Mater., Vol. 40, No 1, 1-30, 1992.

9. Rae, CM.F., Matan, N. and Reed, R.C., "The role of stacking fault shear in the primary creep of [001]oriented single crystal superalloys at $750^{\circ} \mathrm{C}$ and 750 MPa”, Mat. Sci. Engng., A300, 125-134, 2001.

10. Ashby, M.F. and Dyson, B.F., "Creep damage mechanics and micromeehanisms", in Advances in Fracture Research, ed. by S R Valluri et al., (1984) Pergamon Press, 1, 3-30.

11. Basoalto, H.C. and Vermeulen, B., "Modelling of the High Temperature Uniaxial <001> Creep Response of CMSX4", Report QINETIQ/EMEA/TS/CR0701536/1.0, 2007.

12. Dyson, B.F., private communication with author, 2000.

13. Dyson, B.F. and McLean, M., Microstructural Stability of Creep Resistant Alloys for high Temperature Plant Application, Ed. A. Strang et al., The Institute of Materials, 1998.

14. Basoalto, H.C, Sondhi, S.K., Dyson, B.F. and McLean, M., "A generic microstructure-explicit model of creep in nickel-based superalloys", in Superalloys 2004, Ed. Green et al., 897-906, TMS (The Minerals, Metals \& Materials Society), 2004.

15. Sondhi, S.K., Dyson, B.F. and McLean, M., "Tensioncompression creep asymmetry in a turbine disc superalloy: roles of internal stress and thermal ageing", Acta Mater., Vol. 52,7, 1761-1772, 2004.

16. Basoalto, H.C. and Dyson, B.F., to be published.

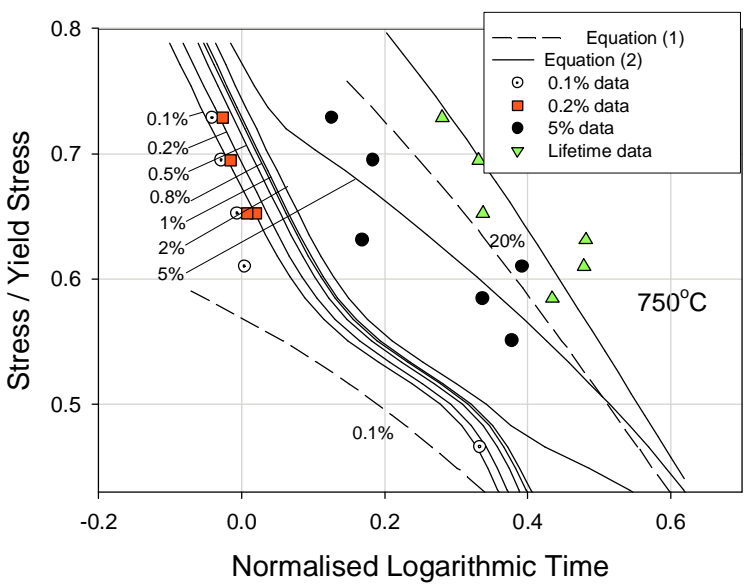

Figure 8 Model predictions of the stress-time contour plots to reach $0.1,0.2,0.5,0.8,1,2,5$ and $20 \%$ creep strain at $750^{\circ} \mathrm{C}$.

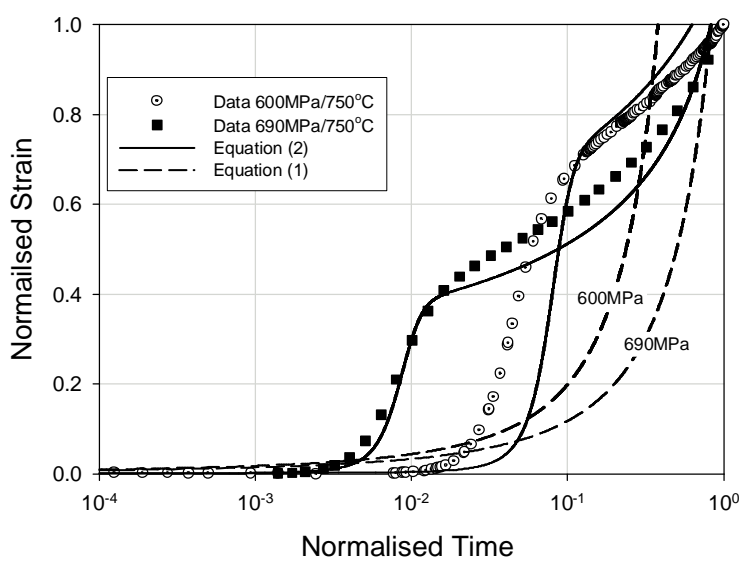

Figure 9 Normalised test data and model predictions of the creep response at $750^{\circ} \mathrm{C}$.

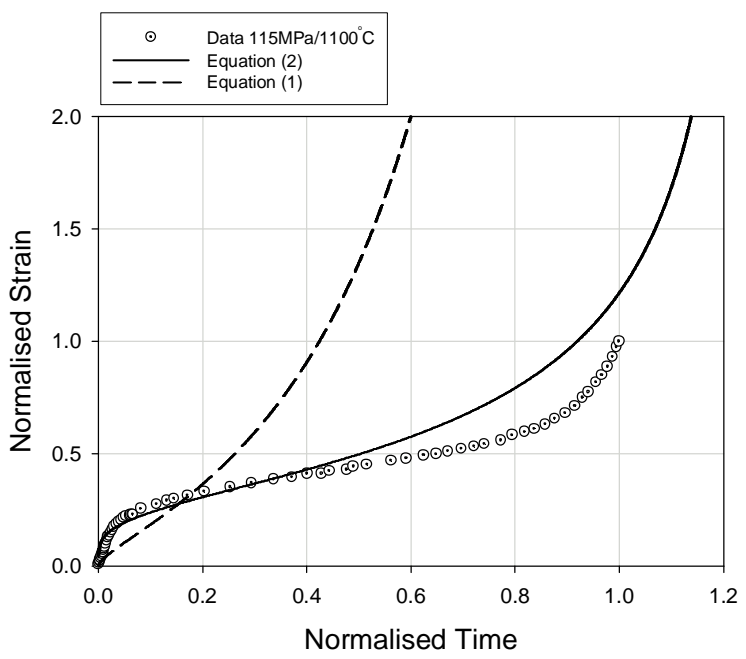

Figure 10 Normalised test data and model predictions of the creep response at $115 \mathrm{MPa} / 1100^{\circ} \mathrm{C}$. 